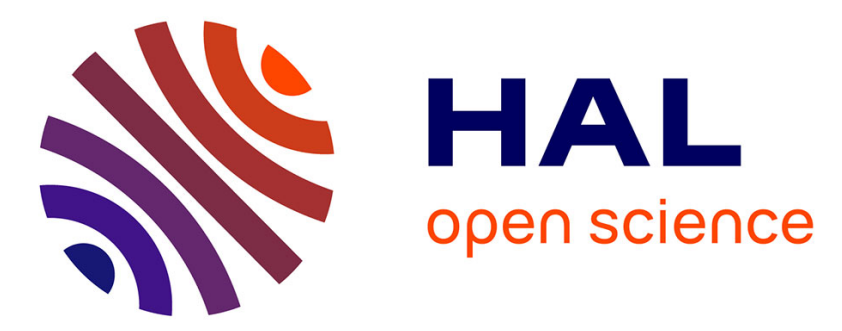

\title{
Un policier français dans l'Empire. Pierre Lefuel, dernier directeur de la Sûreté voltaïque (1959-1960) et pionnier du Service de coopération technique internationale de police (SCTIP) \\ Romain Tiquet
}

\section{To cite this version:}

Romain Tiquet. Un policier français dans l'Empire. Pierre Lefuel, dernier directeur de la Sûreté voltaïque (1959-1960) et pionnier du Service de coopération technique internationale de police (SCTIP). Histoire, économie et société, 2013, Histoire de la force publique aux XIXe et XXe siècles, 2013 (4), pp.49-58. 10.3917/hes.134.0049 . hal-01849259

\author{
HAL Id: hal-01849259 \\ https://hal.science/hal-01849259
}

Submitted on 25 Jul 2018

HAL is a multi-disciplinary open access archive for the deposit and dissemination of scientific research documents, whether they are published or not. The documents may come from teaching and research institutions in France or abroad, or from public or private research centers.
L'archive ouverte pluridisciplinaire HAL, est destinée au dépôt et à la diffusion de documents scientifiques de niveau recherche, publiés ou non, émanant des établissements d'enseignement et de recherche français ou étrangers, des laboratoires publics ou privés. 
UN POLICIER FRANÇAIS DANS L'EMPIRE. PIERRE LEFUEL, DERNIER DIRECTEUR DE LA SÛRETÉ VOLTAÏQUE (1959-1960) ET PIONNIER DU SERVICE DE COOPÉRATION TECHNIQUE INTERNATIONALE DE POLICE (SCTIP)

\author{
Romain Tiquet
}

Armand Colin | Histoire, économie \& société

2013/4 - 32e année

pages 49 à 58

ISSN 0752-5702

Article disponible en ligne à l'adresse:

http://www.cairn.info/revue-histoire-economie-et-societe-2013-4-page-49.htm

Pour citer cet article :

Tiquet Romain, « Un policier français dans l'Empire. Pierre Lefuel, dernier directeur de la Sûreté voltaïque (1959-1960) et pionnier du Service de coopération technique internationale de police (SCTIP) », Histoire, économie \& société, 2013/4 32e année, p. 49-58.

Distribution électronique Cairn.info pour Armand Colin.

(c) Armand Colin. Tous droits réservés pour tous pays.

La reproduction ou représentation de cet article, notamment par photocopie, n'est autorisée que dans les limites des conditions générales d'utilisation du site ou, le cas échéant, des conditions générales de la licence souscrite par votre établissement. Toute autre reproduction ou représentation, en tout ou partie, sous quelque forme et de quelque manière que ce soit, est interdite sauf accord préalable et écrit de l'éditeur, en dehors des cas prévus par la législation en vigueur en France. II est précisé que son stockage dans une base de données est également interdit. 


\title{
Un policier français dans l'Empire. Pierre Lefuel, dernier directeur de la Sûreté voltaïque (1959-1960) et pionnier du Service de coopération technique internationale de police (SCTIP)
}

\author{
Romain Tiquet
}

\section{Résumé}

La trajectoire professionnelle de Pierre Lefuel, dernier directeur de la Sûreté de Haute-Volta (actuel Burkina Faso) avant l'indépendance et pionnier de la création du service de coopération policière, apporte un éclairage original sur l'action policière de la France dans ses anciennes colonies africaines. La carrière de Pierre Lefuel se révèle intéressante à plus d'un titre, car elle met en exergue les choix tactiques de la France pour maintenir sa position de domination en matière de sûreté pendant la période coloniale et postcoloniale.

\section{Abstract}

Focusing on Pierre Lefuel's professionnal trajectory (last Head of colonial police unit in UpperVolta and pionner of police cooperation unit), this paper brings to light an innovative approach on France's police strategies in its former african colonial territories. Pierre Lefuel's career is found to be outstanding since it reveals tactical decisions made by French officials to uphold its domination regarding policing within the colonial and postcolonial period.

« Monsieur Lefuel dirige avec brio une section qui constitue l'ossature même du SCTIP et sa justification ${ }^{1}$. » C'est en ces termes qu'Alfred Diefenbacher, directeur de ce service, décrit en 1971 Pierre Lefuel, alors responsable de la section pédagogique et d'assistance technique ${ }^{2}$. Pierre Lefuel, né le 28 décembre 1921 et décédé le 28 juin 2010, a passé près de trente-sept années au sein de la Police nationale. Il commence sa carrière en 1944, comme

1. Archives Nationales [désormais AN] 19850641 article 69 : Bulletin de note 1971.

2. Je remercie Jean-Pierre Bat et Nicolas Courtin pour leur relecture et leurs conseils et avisés. 
jeune commissaire stagiaire à Senlis, et prend sa retraite, en décembre 1981, avec le grade de contrôleur général de police. Envoyé en Afrique Occidentale Française (AOF) en 1946, il officie d'abord en Côte d'Ivoire puis au Soudan français (actuel Mali) et au Sénégal, pour terminer sa carrière « coloniale » en Haute-Volta (actuel Burkina Faso) en tant que dernier directeur de la Sûreté. Placé à un poste charnière à l'orée des indépendances africaines, il joue un rôle déterminant dans la transmission des services de police voltaïque à l'État indépendant. De retour en France dès 1961, il contribue à créer le SCTIP et prend la tête du service en charge de la formation des policiers et de l'assistance technique. À cet égard, Pierre Lefuel constitue un exemple emblématique de la continuité de la France dans son action policière en Afrique de l'Ouest.

L'organisation des services de police civile reste embryonnaire en AOF jusque dans l'entre-deux-guerres. D'autres autorités policières ont été mises en place dès la fin de la pacification : la gendarmerie coloniale, en 1854, puis les gardes cercles, au début du $\mathrm{xx}^{\mathrm{e}}$ siècle, auxquels s'ajoutent l'administrateur colonial, officier de police judiciaire sous l'autorité du gouverneur, et les chefs traditionnels, chargés de lever les impôts et de la police des marchés. En ce qui concerne la police civile, il faut attendre 1918 pour voir la création d'un service de Sûreté générale de l'AOF, rattaché en 1923 à la direction des services des affaires politiques du gouvernement général. En 1931 sont créés le service central de la sûreté à Dakar et le service des Renseignements généraux (RG). En 1944, tous ces organismes sont englobés dans l'Inspection générale des services de sécurité (IGSS) de l'AOF. Subissant les aléas politiques du territoire - la colonie, créée en 1919, est supprimée en 1932 pour être reconstituée en 1947 -, les services de police voltaïques n'apparaissent qu'en 1949. Même si le dernier-né des territoires de l'AOF abritait déjà des brigades mobiles de sécurité, il faut attendre le mois de décembre 1949 pour qu'un arrêté institue juridiquement une Sûreté ${ }^{3}$. Agissant principalement dans les villes, les services de police se spécialisent dans le renseignement, et la figure du policier colonial, en tant qu'agent de l'État, voit ainsi son champ d'action se délimiter autour du maintien de l'ordre colonial à travers le contrôle et le respect d'un ordre légal.

Le maintien de l'ordre a la particularité d'être à la fois fondamental dans la justification de l'empire et « tout à fait secondaire dans les théorisations sur la domination coloniale ${ }^{4}$. » Alors que l'historiographie anglo-saxonne étudie depuis longtemps le colonial policing ${ }^{5}$, les ouvrages français sur la question restent rares, et l'histoire des polices en situation coloniale dans l'Empire français souffre d'un véritable « trou noir historiographique ${ }^{6}$ » malgré quelques travaux récents ${ }^{7}$.

3. Arrêté n 498/SU du 23 décembre 1949 instituant une « Sûreté territoriale » en Haute-Volta.

4. Emmanuel Blanchard et Joël Glasman, « Le maintien de l'ordre dans l'empire français : une historiographie émergente », dans Maintenir l'ordre colonial. Afrique et Madagascar, XIX ${ }^{e}-X X^{e}$ siècles, dir. Jean-Pierre Bat et Nicolas Courtin, Rennes, PUR, 2012, p. 11.

5. Voir, par exemple, les programmes du Colonial and Postcolonial Policing Group (COPP) de 1'Open University, ainsi qu'Anthony Clayton et David Killingray, Khaki and Blue, Military and Police in British Colonial Africa, Athens, Ohio University Press, 1989; David Killingray et David Anderson, Policing and Decolonisation. Politics, Nationalism and the Police, 1917-1965, Manchester, Manchester University Press, 1991 ; David Killingray et David Omissi (dir.), Guardians of Empire, The Armed Forces of The Colonial Powers c. 1700-1964, Manchester, MUP, 1999 ; Martin Thomas, Violence and colonial order : police, workers and protest in the European colonial empires, 1918-1940, Cambridge, CUP, 2012.

6. Jean-Marc Berlière, « Entre pages blanches et légendes : un corps sans mémoire ? », Pouvoirs, 2002/102, p. 5-15, ici p. 7.

7. Pour une synthèse des travaux français en cours sur les « corps habillés » coloniaux. Jean-Pierre Bat et Nicolas Courtin (dir.), Maintenir l'ordre colonial. Afrique et Madagascar..., op. cit. 
Cette étude analyse, sous un angle original, l'action policière de la France en Afrique au travers de la trajectoire professionnelle de Pierre Lefuel. Elle se fonde sur son dossier de carrière, qui se révèle être un véritable outil de recherche en éclairant les pratiques professionnelles propres à l'institution policière ${ }^{8}$. Appuyée sur un entretien oral réalisé en mai 2010 et sur des sources d'archives, la découverte du parcours de ce policier hors-norme apprend beaucoup sur les logiques et le fonctionnement de la police coloniale et du SCTIP.

\section{Un policier dans l'empire : le cursus honorum colonial}

\section{Du commissaire stagiaire à Senlis (juin 1944) à la direction de la Sûreté voltaïque (juin 1959)}

Qu'est-ce qui pousse le jeune commissaire Lefuel à partir pour l'AOF, deux ans à peine après le début de sa carrière ? Sa réponse reste assez lapidaire : "Comme on gagnait pas beaucoup et que j'avais un tas de collègues qui partaient en Indochine, j'ai rencontré quelqu'un qui travaillait en Afrique noire. J'ai fait une demande et je suis parti avec ma femme début $1946^{9}$. » Un événement passé sous silence par l'intéressé, mais révélé par son dossier de carrière, nuance quelque peu son affirmation. Fraîchement diplômé de l'école de police, il débute sa carrière à Senlis, le 21 juin 1944, en tant que commissaire stagiaire à la sécurité publique (SP). Son dossier indique que, très vite, ses relations avec le maire de Senlis se dégradent, le maire accusant le jeune commissaire de « traîner avec des mauvais garçons » et d'empêcher les agents de police de s'acquitter de leur fonction ${ }^{10}$ ! Le magistrat demande donc sa mise à la disposition de l'administration centrale et la nomination d'un autre commissaire. Lefuel est alors envoyé, en octobre 1945, au commissariat d'arrondissement de Saint-Quentin, à la direction de la SP. Il est donc probable que ses ennuis relationnels, ajoutés à des opportunités de carrière plus intéressantes en AOF, l'ont poussé à quitter la France pour l'Afrique noire au début de l'année 1946. La possibilité de gravir plus rapidement les échelons de la hiérarchie et la perspective d'un meilleur salaire sont d'ailleurs les principales motivations qui poussent les policiers français à partir aux colonies en début de carrière.

Initialement affecté à Dakar, où il connaît le procureur général, il apprend, à son arrivée au Sénégal, qu'il prend la tête du commissariat central d'Abidjan, en Côte d'Ivoire ${ }^{11}$. Simple commissaire d'arrondissement dans une ville française de taille moyenne, Lefuel est propulsé, après à peine deux ans de carrière, à la tête du commissariat urbain de la première ville de la colonie. Il détient alors un véritable poste à responsabilité, d'autant plus que la ville coloniale constitue le centre névralgique des activités policières. Lieux des décisions militaires, politiques et économiques depuis la pacification, les villes coloniales doivent être envisagées comme des « espaces de sécurité » au sens foucaldien du terme, c'est-à-dire un espace où doit s'imposer la sécurité ${ }^{12}$. On assiste alors à une centralisation

8. Sur la richesse de l'utilisation des dossiers de carrière : Aude Béliard et Emilie Biland, « Enquêter à partir de dossiers personnels. Une ethnographie des relations entre institutions et individus », Genèses, 2008/70, p. 106-119. Pour les carrières policières africaines, Joël Glasman, « Penser les intermédiaires coloniaux : note sur les dossiers de carrière de la police au Togo », History in Africa, 2010/37, p. 51-81.

9. Entretien avec Pierre Lefuel, Cannes : 5 mai 2010.

10. AN 19850641 article 69 : Lettre du commissaire principal de Senlis agrafée au bulletin de note de l'année 1945.

11. Entretien Pierre Lefuel, Cannes : 5 mai 2010.

12. Michel Foucault, Sécurité, territoire, population : cours au Collège de France (1977-1978), Paris, Gallimard-Seuil, 2004. 
des forces de police civiles en ville, alors que la gendarmerie et les gardes cercles officient plutôt dans les campagnes ${ }^{13}$.

En poste à Abidjan jusqu'en 1952, Pierre Lefuel est muté, en $1953^{14}$, au Soudan français, en tant que commissaire central de la ville de Bamako. Reconnu par sa hiérarchie comme un policier de grande qualité, il est affecté, en 1956, à la Sûreté générale de Dakar en tant qu'adjoint au chef de la Sûreté. Appelé, en 1959, par le nouveau gouverneur de la Haute-Volta, Paul Masson, il termine sa carrière « coloniale » à la tête des services de police voltaïques à l'aube des indépendances.

\section{Policier métropolitain en colonie : "l'œeil du pouvoir »?}

Une métaphore récurrente assimile souvent la police à «l'œil du pouvoir ». C'est d'abord par l'œil que la police semble acquérir son savoir, et par son regard qu'elle maintient l'ordre et assure la sécurité ${ }^{15}$. La priorité policière va, dans les années 1940-1950, à l'organisation du service de renseignement et au maintien de l'ordre politique ${ }^{16}$. La création des services de police en $\mathrm{AOF}$ a donc un objectif clair : mieux contrôler les territoires et lutter contre les mouvements politiques dissidents qui tendent à se développer.

Dans le cadre de la Haute-Volta, tout l'appareil de surveillance mis en place par les RG permet à la Sûreté locale de contrôler, ou au moins de suivre, les évolutions politiques, économiques et sociales de la colonie. Bien que l'activité policière soit cantonnée aux centres urbains (Ouagadougou et Bobo-Dioulasso pour la Haute-Volta), la Sûreté peut compter sur des brigades mobiles de renseignement aux compétences étendues. Placées sous l'autorité du chef de la Sûreté, leur principale mission est de renseigner sur la vie politique du territoire. Comme le rappelle Albert Mouragues, ancien gouverneur de la Haute-Volta, les brigades mobiles doivent être « l'œil et l'oreille du gouverneur ${ }^{17}$. »

La surveillance, dans le cadre colonial, est plus qu'une fonction de la police : un mode de gouvernement. La police ne détient d'ailleurs pas le monopole du renseignement, puisque toute l'administration informe. Policiers, gendarmes, militaires, administrateurs locaux, chefs traditionnels et même missionnaires : un véritable maillage institutionnel assure un contrôle indispensable à un objectif essentiel, le maintien de l'ordre. Essentiel, car, comme le soulignent Emmanuel Blanchard et Joël Glasman, maintenir l'ordre « est un problème qui engage l'ensemble du projet colonial. [Ce] n'est pas, en colonie, une question technique, c'est la condition sine qua non de l'existence d'une société coloniale ${ }^{18}$. »

Cette définition du maintien de l'ordre colonial ne présume en rien le succès de cette prétention. En Haute-Volta, même si quatre brigades mobiles sont en théorie présentes sur le territoire, seule la brigade de Bobo-Dioulasso fonctionne dans les années 1950 pour

13. Pour une synthèse détaillée à ce sujet, Bénédicte Brunet-La Ruche, « "Discipliner les villes coloniales" : la police et l'ordre urbain au Dahomey pendant l'entre-deux-guerres », Criminocorpus, revue hypermédia, Histoire de la police, Articles, mis en ligne le 13 janvier 2012, consulté le 11 juillet 2013.

14. Les policiers français en $\mathrm{AOF}$ ont le droit à six mois de congés tous les deux ans de service.

15. Hélène L'Heuillet, Basse politique, haute police : une approche philosophique et historique, Paris, Fayart, 2001, p. 213.

16. Laurent Fourchard, De la ville coloniale à la cour africaine : espaces, pouvoirs et sociétés à Ouagadougou et Bobo-Dioulasso, Haute-Volta : fin XIX siècle-1960, Paris, L'Harmattan, 2001, p. 278.

17. Archives nationales de Ouagadougou [désormais ANO], 3 V 10 : Conférence des administrateurs coloniaux en AOF (1949-1954), exposé sur la situation générale de la police en AOF lors de la conférence des gouverneurs coloniaux, 23 juin 1949.

18. Emmanuel Blanchard et Joël Glasman, op. cit., p. 13. 
lutter contre le Rassemblement Démocratique Africain (RDA) ${ }^{19}$, très présent dans le sud du territoire. En Côte d'Ivoire, le budget et la formation des policiers faisant défaut, Pierre Lefuel explique qu'il a passé plus de temps à organiser et discipliner ses effectifs qu'à réaliser les missions qui lui sont dévolues :

À Abidjan, j'avais essayé d'organiser des patrouilles mais c'était pas possible, les gars s'endormaient en route. Ils étaient dotés d'un mousqueton pour qu'ils effraient un peu les voleurs, mais, la nuit, quand je me levais et que j'allais inspecter, je les trouvais endormis avec le mousqueton à côtée ${ }^{20}$.

Ces exemples montrent le caractère empirique de l'action policière et ses limites. Loin de l'image d'une police véritable gardienne de l'empire, le travail des policiers coloniaux reste avant tout le fruit de « bricolages » et d'adaptations aux situations locales.

\section{La double casquette de Lefuel, résultat d'une double transmission des prérogatives policières}

\section{Loi-cadre et Communauté franco-africaine : un legs partiel et partial}

La loi-cadre Deferre de 1956 marque un changement de cap dans la gouvernementalité coloniale, au sein de l'Union française mise en place en 1946, en insufflant deux processus politiques : une politique de déconcentration, qui transfère aux chefs de territoires de nombreux domaines anciennement régis par le pouvoir métropolitain, et une politique de décentralisation, qui conduit à créer des conseils de gouvernement et des assemblées locales dotés de pouvoirs réglementaires étendus. Dans un contexte de flou administratif, cette réforme entraîne de nombreux débats internes quant à la gestion de l'organisation policière ${ }^{21}$. La loi distingue des services d'État, « chargés de la gestion des intérêts de l'État », et des services territoriaux, « chargés de la gestion des intérêts des territoires ${ }^{22}$ ». Le décret d'application précise que les services de police administrative constituent des services d'État, à l'exception des services de police municipale et rurale. Cette définition du périmètre de la police d'État identifie bien les autres services de police comme de simples services territoriaux. Le décret marque une évolution importante, puisque le Hautcommissaire n'a plus le pouvoir d'organiser, ni de payer, les services de police municipale et rurale, qui deviennent à la charge du conseil de gouvernement et de l'assemblée territoriale.

Le décret d'application ne délimitant pas le cadre d'intervention des services territoriaux, l'autorité coloniale s'empresse de le définir d'une manière restrictive afin de garder la main sur les fonctions policières dominantes $(\mathrm{RG}$, sécurité du territoire et police judiciaire). Les autorités françaises vident ainsi de leur substance les pouvoirs légués aux autorités locales afin que le pouvoir colonial garde le monopole de l'organisation policière. L'autonomie interne chère à la loi-cadre est loin d'être acquise en matière de police.

Après le remplacement, en 1958, de l'Union française par la Communauté française, à laquelle les pays africains peuvent choisir de ne pas adhérer, les chefs de gouvernement

19. Le RDA est une ancienne fédération de partis politiques africains fondée à l'issue du Congrès de Bamako (18-21 octobre 1946). Elle est considérée comme le premier mouvement politique panafricain.

20. Entretien avec Pierre Lefuel, Cannes : 2 mai 2010.

21. Pour une étude plus détaillée : Romain Tiquet, «D'un État à l'autre, la stratégie du Guépard policier : transfert total ou legs partiel des pouvoirs de police en Haute-Volta (1949-1960) ? », dans Maintenir l'ordre colonial..., op. cit., p. 133-142.

22. Article 3 de la loi n 56-619 du 23 juin 1956. JORF, 24 juin 1956. 
des États de l'AOF procèdent, via un organisme de transferts et de liquidations présidé par le gouverneur, à la transmission d'une grande partie des compétences de sûreté aux États membres $^{23}$. Ces États acquièrent dès lors des pouvoirs en matière de police judiciaire et de police administrative. Ils peuvent désormais traquer les crimes et délits, contrôler les trafics d'armes, lutter contre le banditisme, établir des interdictions de séjour et procéder à des enquêtes administratives. Avec ces transferts de fonctions essentiellement répressives, le pouvoir colonial laisse aux représentants des nouveaux États autonomes le soin d'organiser la surveillance des mouvements politiques dont ils ont souvent été les initiateurs et qui les ont portés au pouvoir.

Le pouvoir colonial garde en revanche la haute main sur diverses fonctions régaliennes touchant directement à la sûreté de la Communauté. Il conserve le pôle « ImmigrationÉmigration », permettant à l'État métropolitain de contrôler les mouvements de populations sur tous les territoires de la Communauté, et il garde aussi le contrôle « de la sûreté extérieure et [de la] Défense nationale ${ }^{24} »$. La Communauté se décharge des fonctions répressives de la police pour se concentrer essentiellement sur la défense et la surveillance du territoire.

En transférant les opérations de répression et de maintien de l'ordre aux élites installées au pouvoir, la France institue ces mêmes élites comme les nouvelles garantes de la permanence de la domination de la métropole. Si la loi-cadre et la Communauté ont mis en œuvre le principe d'autonomie des territoires, le transfert des compétences policières a été organisé en fonction du choix, politique, de sauvegarder les intérêts métropolitains.

\section{Le jeu d'équilibriste de Lefuel, directeur de la Sûreté et directeur du SSEC}

C'est dans ce contexte de tâtonnements institutionnels et de transferts partiels des prérogatives policières que Pierre Lefuel est appelé, en juin 1959, à la direction de la Sûreté voltaïque. Cette affectation n'a rien d'un hasard, puisque Lefuel est nommé personnellement par le gouverneur Paul Masson, qu'il a rencontré à Bamako, où il était commissaire, et Masson, gouverneur. Ce poste revêt un caractère éminemment politique, et Masson compte sur le commissaire et sur ses services pour assurer une retraite en bon ordre. De plus, Lefuel est chargé de la direction locale du service de sécurité extérieure de la Communauté (SSEC).

Le SSEC est créé, en mai 1959, à l'initiative du préfet Jean Poitevin, en raison du choix par la Guinée de son indépendance, en septembre 1958, qui renforce l'idée du pouvoir colonial de maintenir la cohésion de la Communauté en luttant contre toute menée subversive. Institué dans un climat de plus en plus marqué par les mouvements d'opposition, le SSEC se concentre essentiellement sur la surveillance des frontières et procède à la recherche et à la répression des atteintes à la sécurité extérieure de la Communauté. Il joue le rôle, à l'égard des services extérieurs, d'un « véritable bureau de renseignement ${ }^{25}$ » et permet à la France de maintenir sa surveillance et son influence sur la Communauté.

Le SSEC souhaite avant tout se présenter comme un organe de liaison entre les services parisiens (direction centrale du SSEC, mais aussi DST et SDECE) et les services locaux

23. Arrêté n 3101/IGAA du 31 mars 1959, qui porte «transfert de compétences détenues par le Hautcommissariat général (direction des services de sécurité) aux Républiques de Côte d'Ivoire, Dahomey, HauteVolta, Mauritanie, Niger, Sénégal, Soudan ».

24. Article 2 de l'arrêté du 31 mars 1959.

25. Centre d'archives diplomatiques de Nantes [désormais CADN], 184 PO/1/473, Dakar, Ambassade : services annexes, délégation du SCTIP (personnel, dossier général, budget), correspondances, instruction générale à l'intention des directeurs des services de sécurité extérieure, le 15 janvier 1960. 
(délégations du SSEC et services de polices locaux). Cette précision est importante, car elle est caractéristique du jeu d'équilibriste entrepris par les autorités françaises, qui souhaitent garder le contrôle des fonctions souveraines de police tout en essayant de ne pas froisser les exécutifs locaux. Le SSEC dispose alors d'une direction centrale, relevant du Premier ministre, et de services locaux dans chacun des États membres. Ils sont théoriquement divisés en sections et composés de brigades mobiles, mais cette organisation reste théorique, car les services de police sont pour la plupart en sous-effectifs. En HauteVolta, la direction locale du SSEC est dirigée par Pierre Lefuel et représentée par un agent africain à Ouagadougou pour le renseignement et par un autre à Bobo-Dioulasso ${ }^{26}$.

Dans un contexte où les exécutifs locaux demandent de plus en plus d'autonomie et de souveraineté, la double casquette de Lefuel met les services de police dans une position plus qu'ambiguë. Lefuel dépend en théorie de l'exécutif voltaïque en tant que directeur de la Sûreté, mais il a à sa charge la direction locale du SSEC, véritable service de renseignement pour le compte de la puissance coloniale française ! Paul Masson résume assez bien cette ambiguiité en comparant la position française en Haute-Volta à celle d' " un trapéziste qui se balance sur une corde raide, chacun se demandant ce que va faire l'artiste et comment il va se rétablir dans ce périlleux exercice sans filet ${ }^{27}{ }$. La situation perdure jusqu'à l'indépendance, signée le 5 août 1960. Pierre Lefuel est remplacé quelques mois après par le premier directeur africain de la Sûreté voltaïque, Michel Compaoré, que le président Maurice Yameogo, mécontent de son travail, remplace, en 1963, par un officier.

\section{Lefuel et la mise en place du SCTIP : une coopération fidèle au modèle ?}

\section{Le SSEC est mort, vive le SCTIP!}

Même si, en théorie, le SSEC se présente comme la clef de voûte de la sûreté dans la Communauté, en pratique, son action est très limitée. Les indépendances africaines finissent par achever ce service, qui ne peut même pas effectuer ses missions, puisqu' aucun texte juridique n'est venu les définir ${ }^{28}$. Lefuel donne sa version de l'évolution de la situation :

[...] Comme c'était devenu intenable, le SSEC ne dura pas longtemps, et le ministère de l'Intérieur à Paris a créé le Service de coopération technique internationale de police. J'ai été un des fondateurs du SCTIP, car, en fait, on m'a dit : vous venez d'Afrique, vous connaissez le coin, vous venez ${ }^{29}$.

La France crée le SCTIP ${ }^{30}$ par décret interministériel du 14 décembre $1961^{31}$. Cet organisme se développe dans la continuité du SSEC, dans un contexte où la France se

26. Cet agent cumule la fonction d'agent du SSEC et celle d'inspecteur à la brigade mobile de l'Ouest-Volta. Pour un portrait de carrière de cet inspecteur, Romain Tiquet, «Hubert Kho, premier Africain de la Sûreté coloniale voltaïque (1950-1963) », dans Maintenir l'ordre colonial..., op. cit., p. 199-207.

27. Paul Masson, «Évolution de la Haute-Volta vers l'Indépendance et proclamation de l'Indépendance (1958-1960) », dans La Haute-Volta coloniale : témoignages, recherches, regards, Paris, Khartala, 1995, p. 497.

28. CADN, 184 PO/1/473, Dakar ambassade : Services annexes délégation du SCTIP (personnel, dossier général, budget), SSEC et SCTIP, Secrétaire d'État aux relations avec les États de la Communauté à Monsieur le Haut Représentant de France à Dakar à propos de la suppression du SSEC, le 2 mai 1961.

29. Entretien avec Pierre Lefuel, Cannes : 5 mai 2010.

30. Pour plus de détails sur le passage du SSEC au SCTIP, Jean-Pierre Bat, « 1961 : la naissance du SCTIP », Société française d'histoire de la police, article en ligne, consulté le 12 juillet 2013.

31. Décret n 61-1373 du 14 décembre 1961 portant création au ministère de l'Intérieur d'un Service de coopération technique internationale de police, JORF, 16 décembre 1961. 
préoccupe avant tout de maintenir son influence en proposant ses services (assistance technique et formation) aux nouveaux États africains indépendants. Son originalité réside dans le fait qu'il dépend du ministère de l'Intérieur, et non du ministère de la Coopération. C'est l'une des innovations majeures, car il est chapeauté par un préfet et jouit d'une grande autonomie, contrairement aux lourdeurs administratives du ministère de la Coopération.

Renaissant des cendres du SSEC, le SCTIP devait-il intégrer le renseignement dans ses missions à l'étranger? Dans une lettre destinée au Haut-Représentant de la France à Dakar, le secrétaire d'État aux relations avec les États de la Communauté élude la question :

[...] Finalement, il a été décidé de ne pas inclure cette recherche [de renseignement] dans la mission du SCTIP, car elle risquerait de le rendre vulnérable et suspect aux yeux des États. Il n'en reste pas moins que les renseignements que le service recueillera, même sans les rechercher, peuvent et doivent être utilisés ${ }^{32}$.

Lefuel corrobore cette tactique : "C'était pas officiel mais à titre confidentiel ; chaque délégué du SCTIP dans les territoires faisait du renseignement comme il le pouvait ${ }^{33}$. »

Le SCTIP, qui ne représente pas beaucoup de personnes au début des années 1960, est essentiellement composé d'anciens policiers coloniaux qui ont pour la plupart travaillé pour le SSEC. Une note du 2 mai 1961 insiste sur l'utilisation « du personnel actuellement en service au $\mathrm{SSEC}^{34}$ » pour mettre sur pied les antennes locales du SCTIP. La France a, en quelque sorte, recyclé ses anciens policiers coloniaux, des sûretés locales au SCTIP en passant par le SSEC. En faisant appel à ce vivier, le SCTIP souhaite conserver la maîtrise de la formation et de l'assistance en matière de police pour tenter de refonder, à terme, une sorte de « pacte colonial », c'est-à-dire un système de coopération policière dont la France tirerait la grande majorité des avantages. Françoise Lamborelle, fonctionnaire au SCTIP dans les premières années de sa création, résume bien la stratégie : «Nous étions un peu l'arrière-garde d'une France colonialiste et nous sommes devenus l'un des fers de lance de la politique de coopération internationale de notre pays ${ }^{35}$. »

L'objectif principal de la coopération policière est double : offrir un service au lendemain des indépendances africaines, en aidant les nouvelles républiques à se doter d'une indispensable force publique, et placer auprès des nouveaux chefs d'Etat des hommes sûrs, capables de mettre en place des réseaux francophiles.

\section{Lefuel et la formation des policiers : héritages coloniaux et continuité des pratiques}

Les polices africaines issues des anciens territoires coloniaux rencontrent un problème majeur : le manque de formation de leur personnel. La hiérarchie policière n'est pas prête à prendre du jour au lendemain les rênes des nouveaux corps. Les sûretés se retrouvent dans l'incapacité de mettre en place des programmes de formation professionnelle garantissant le fonctionnement correct de leurs services. Le SCTIP s'impose dès lors comme un acteur central, voire indispensable, pour l'instruction des personnels africains. Pour les gardiens de la paix, une formation est assurée sur place par les assistants techniques français. Pour les personnels encadrants (officiers, inspecteurs et commissaires de police), le SCTIP

32. CADN, 184 PO/1/473, déjà cité, p. 3.

33. Entretien Pierre Lefuel, Cannes : 5 mai 2010.

34. CADN, 184 PO/1/473, déjà cité, p. 3.

35. Revue Équinoxe, bulletin interne du SCTIP, 2002/18, publication du ministère de l'Intérieur, p. 3. 
propose de les incorporer aux promotions de l'école de Saint-Cyr-au-Mont-d'Or ${ }^{36}$ sans différence de cursus.

Le service rencontre quelques difficultés d'adaptation au début de son existence, car il tente de répondre au traditionnel «Que fait la police ? », alors que la question centrale dans les États africains était plutôt «Qui fait la police ? ». Dans les premières années, le SCTIP calque la formation française en Afrique pour parer au plus urgent et éviter toute improvisation dangereuse. Ensuite, une prise de recul permet d'orienter autrement les formations. Les policiers africains appelés à de hautes responsabilités sans avoir gravi les différents échelons hiérarchiques reçoivent un enseignement essentiellement technique et pratique.

De par son expérience passée en Afrique de l'Ouest et ses capacités d'encadrement et de pédagogie, Lefuel devient le véritable chef de file du SCTIP. Nommé dès 1962 responsable de la section pédagogique et d'assistance technique, il organise de toutes pièces des programmes et des établissements de formation pour les États africains ${ }^{37}$ : «Au SCTIP, j'ai dû créer 13 ou 15 écoles de police surtout en AOF [sic] ; ça a été une partie passionnante pour moi, car je créais les écoles, je formais les instructeurs à Paris, je me suis impliqué dans les méthodes audiovisuelles en réalisant des films de formation ${ }^{38}$ ». Apprécié par la grande majorité des États africains pour son professionnalisme, Pierre Lefuel incarne tout au long de sa carrière au SCTIP la réussite de ce service, comme le souligne son bulletin de note en 1965 : «Chargé de la formation professionnelle des polices africaines, M. Lefuel a mis au point, en quelques années, des ouvrages et des méthodes d'enseignement dont l'efficacité et l'intérêt ont très largement contribué au succès des missions du SCTIP dans les pays étrangers ${ }^{39}$ ». Reconnu pour ses qualités pédagogiques, il est invité, en 1972, par la direction du SCTIP, à postuler au poste de directeur de l'école nationale de police de Saint-Cyr-au-Mont-d'Or, mais sa candidature n'est pas retenue ${ }^{40}$. Lefuel et son service ont largement contribué à l'imprégnation française de la formation des personnels policiers africains. Le SCTIP, tant par ses missions que par le personnel employé, a orienté les pratiques des futurs policiers selon des schémas professionnels et procéduriers propres à la police française, et proches des anciens choix du pouvoir colonial.

Après plus de quinze années passées au SCTIP, Pierre Lefuel prend en charge, en 1977, la sous-direction de la formation à la Police nationale jusqu'en 1981, date à laquelle il est admis à la retraite. Ce policier, qui a donné tout au long de sa carrière entière satisfaction à sa hiérarchie, a marqué de son poids, tant personnel qu'institutionnel, l'action policière française. Ses trajectoires professionnelles sont intéressantes à plus d'un titre, car elles mettent en exergue les choix tactiques de la France pour maintenir sa position dominante en matière de sûreté pendant la période coloniale et postcoloniale. Elles démontrent par ailleurs la transition professionnelle réussie d'un policier qui a su utiliser son expérience passée pour devenir l'un des piliers du SCTIP.

La carrière de Lefuel est caractéristique d'une période charnière, où le pouvoir colonial souhaitait s'adapter à la situation politique mouvante au sein des colonies afin de défendre jusqu'au dernier moment ses intérêts. La mise en place de la coopération policière et de la

36. Les liens que cette école a tissés avec les policiers africains sont encore très prégnants aujourd'hui.

37. Il soutiendra plus tard un doctorat de droit sur l'organisation de la formation professionnelle des policiers en Afrique. Pierre Lefuel, Étude d'un système d'enseignement professionnel par les médias dans les pays de l'Afrique francophone, doctorat, Paris XIII, 1978.

38. Entretien avec Pierre Lefuel, Cannes : 5 mai 2010.

39. AN, 19850641, article 69 : Bulletin de note, 1965.

40. Ibid. : Bulletin de note, 1973 
formation professionnelle des policiers africains au lendemain des indépendances a modelé les structures mentales et réussi à positionner le SCTIP comme un service permettant à l'ancienne métropole de conserver son influence dans son « pré carré » africain ${ }^{41}$.

UNIVERSITÉ HuMBOLDT, BERLIN

41. Jean-Pierre Bat, Le Syndrome Foccart. La politique française en Afrique de 1959 à nos jours, Paris, Gallimard, 2012. 